\begin{tabular}{|c|c|}
\hline Title & High-Density Liquid W ater at a W ater-Ice Interface \\
\hline Author(s) & $\begin{array}{l}\text { Niinomi, Hiromasa; Y amazaki, T omoya; Nada, Hiroki; Hama, Tetsuya; Kouchi, A kira; Okada, Junpei T.; Nozawa, Jun; } \\
\text { Uda, Satoshi; Kimura, Y uki }\end{array}$ \\
\hline Citation & $\begin{array}{l}\text { Journal of physical chemistry letters, 11(16), 6779-6784 } \\
\text { https://doi.org/10.1021/acs.jpclett.0c01907 }\end{array}$ \\
\hline Issue Date & $2020-08-20$ \\
\hline Doc URL & http:/hdl.handle.net/2115/82514 \\
\hline Rights & $\begin{array}{l}\text { This document is the A ccepted Manuscript version of a Published Work that appeared in final form in Journal of } \\
\text { physical chemistry letters, copyright @ @ A merican Chemical Society after peer review and technical editing by the } \\
\text { publisher. To access the final edited and published work see https://pubs.acs.org/doi/10.1021/acs.jpclett.0c01907. }\end{array}$ \\
\hline Tyре & article (author version) \\
\hline File Information & Journal of physical chemistry letters_11(16)_6779-6784.pdf \\
\hline
\end{tabular}

Instructions for use 


\title{
High-Density Liquid Water at a Water-Ice Interface
}

AUTHOR NAMES.

Hiromasa Niinomi ${ }^{* \dagger}$, Tomoya Yamazaki ${ }^{*}$,Hiroki Nada ${ }^{\S}$, Tetsuya Hama ${ }^{\text {II }}$, Akira Kouchi ${ }^{*}$, Junpei T. Okada ${ }^{\dagger}$, Jun Nozawa $a^{\dagger}$ Satoshi Uda $a^{\dagger}$ and Yuki Kimura*t

\author{
AUTHOR ADDRESSES \\ $\dagger$ Institute for Materials Research, Tohoku University, Sendai, Miyagi, 980-8577, Japan \\ Institute of Low Temperature Science, Hokkaido University, Kita-19, Nishi-8, Kita-ku, \\ Sapporo, 060-0819, Japan \\ ${ }^{\S}$ National Institute of Advanced Industrial Science and Technology (AIST), 16-1 Onogawa, \\ Tsukuba 305-8569, Japan \\ "Komaba Institute for Science, the University of Tokyo, 3-8-1 Komaba, Meguro, Tokyo 153- \\ 8902, Japan
}

* Corresponding Authors: Hiromasa Niinomi (HN) and Yuki Kimura (YK)

Email: h.niinomi@imr.tohoku.ac.jp (HN), ykimura@lowtem.hokudai.ac.jp (YK) 


\begin{abstract}
Because ice surfaces catalyse various key chemical reactions impacting nature and human life, the structure and dynamics of interfacial layers between water vapor and ice have been extensively debated with attention to the quasi-liquid layer. Other interfaces between liquid water and ice remain relatively underexplored, despite their importance and abundance on the Earth and icy extraterrestrial bodies. By in situ optical microscopy, we found that a high-density liquid layer, distinguishable from bulk water, formed at the interface between water and high-pressure ice III or VI when they were grown or melted in a sapphire anvil cell. The liquid layer showed a bicontinuous pattern, indicating that immiscible waters with distinct structures were separated on the interfaces in a similar manner to liquid-liquid phase separation through spinodal decomposition. Our observations not only provide a novel opportunity to explore ice surfaces, but also give insight into the two kinds of structured water.
\end{abstract}




\section{TOC Graphic}

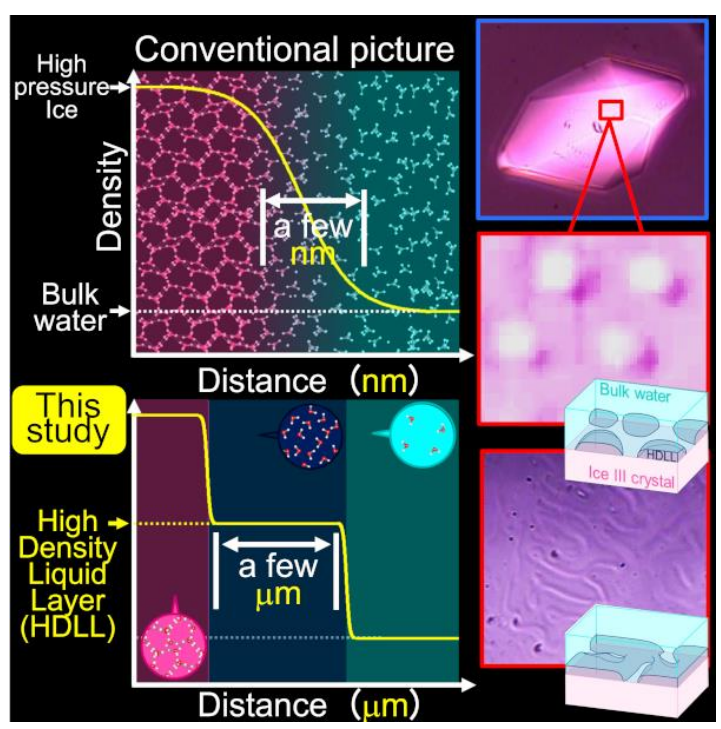

Keywords: High-pressure ice, Ice-water interface, Liquid structure, High density liquid layer, Liquid immiscibility, Bicontinuous pattern, Crystallization from melt. 


\section{INTRODUCTION}

Ice is an abundant material on the Earth, and its surface plays a crucial role in controlling and catalysing key chemical reactions governing the global climate $^{1}$, geological phenomena ${ }^{2}$, biological processes ${ }^{3}$, and the nurture of aerosols ${ }^{4}$, among others. It is well known that a wide variety of chemical reactions are governed by the structure and dynamics of interfacial layers on ice surfaces ${ }^{5,6}$. The nature of the interface between water vapor and ice Ih has been actively debated in the context of a quasi-liquid layer (QLL) resulting from surface premelting, as predicted by Michael Faraday in $1842^{7}$. Although the properties of the QLL are still a matter of active debate, numerous studies have unravelled many of its structural and dynamic aspects that are distinct from those of liquid water ${ }^{5,8-14}$. These active debates have highlighted the fact that ice surfaces are rich, not only in undiscovered phenomena governing key but obscure chemical processes, but also in their underlying physics, which is important for materials science in relation to surface premelting $^{15}$. It is indisputable, therefore, that the elucidation of surface phenomena on ice is highly desirable for a broad range of scientific fields.

Other interface between liquid water and ice that are abundant on the Earth and on icy extraterrestrial bodies remain relatively underexplored, despite having an importance comparable with that of the water vapor-ice interface ${ }^{16}$. Numerical simulations suggest that ice continuously loses its structure within, at most, a few nanometres of an interfacial layer ${ }^{17-19}$, regardless of the polymorphs ${ }^{20}$. Experimental reports concerning the nature of water-ice interfacial layers are limited because of the high degree of technical difficulty arising from the scale of these interfacial layers and the similarity of properties between water and ice. Nevertheless, some light-scattering experiments have suggested the occurrence of wavelength-scale density fluctuations in a thin

interfacial layer with micron scale thickness between liquid water and growing ice $\mathrm{Ih}^{21-24}$. The 
physical pictures emerging from simulations and from experiments remain unverified with each other, and the nature of the layer remains an open question, highlighting a lack of clarity regarding the actual physical nature of the interface. We have discovered, by simple in situ optical microscopy, that a macroscopic interfacial liquid layer, discretely separated from bulk water, forms on the surface of growing or melting high-pressure ices III and VI in bulk water [Figure $1^{25}$; see also METHODS].

\section{RESULTS AND DISCUSSION}

\section{Formation of a liquid layer separated from bulk water.}

An ice III crystal with a rounded shape developed facets on compression in bulk liquid water in a sapphire anvil cell at $-20^{\circ} \mathrm{C}$ (Figure $2 \mathrm{~A} 0 \mathrm{~s}$ and $1 \mathrm{~s}$ ). Continuous pressurization increased the size of the crystal while maintaining its faceted crystal shape (Figure 2 A $1 \mathrm{~s}$ and $3 \mathrm{~s}$ ). The surface morphology of one of the crystal faces fluctuated in a wavelike manner during crystal growth (Figure 2 A $10 s-13$ s, C a and b), suggesting that the surface of the growing ice exhibited fluidity. On the other hand, slight depressurization after growth of the crystal caused the formation of numerous partially wetting liquid droplets on the crystal surfaces (Figure 2 A $13 \mathrm{~s}, 51.5 \mathrm{~s}, \mathbf{C ~ b}-\mathrm{d}$, and $\mathbf{B}$ right). These liquid droplets repeatedly appeared, disappeared, and moved around the surface, and they were able to coexist with the above-mentioned fluctuating surface (Figure $2 \mathbf{A}$ $13 \mathrm{~s}$ and $\mathbf{C} \mathrm{b}$ ). The droplets coexisting with the fluctuated surface showed a different contrast from that produced by asperities on the fluctuating surface, allowing us to deduce that the morphology of the fluctuating surface is similar to that of a thin liquid layer with concave holes (Figure 2 B left). It must be emphasized that the liquid that separated from the surrounding bulk water with a 
clear interface existed at the interface between the bulk water and the ice III crystal, and that this had had a significant lifetime observable by conventional in situ optical microscopy.

\section{Universality of the liquid layer formation.}

The formation of an interfacial liquid layer distinct from bulk water on the ice III crystal raised the question of whether the formation of such the layer is a universal phenomenon regardless of the polymorph. To investigate this question, we used an optical microscope with a Fizeau interferometer to make in situ observations of the crystal growth of ice VI, a phase that is stable at ambient temperatures and high pressures [See METHODS and Supporting Information (SI) Figure S1]. These observations with the interferometer showed that a thin liquid layer, distinguishable from bulk water, covered the ice VI surface (Figure $3 \mathbf{A}-\mathbf{I}$ ) whereas this liquid layer could not be observed by simple bright-field microscopy (Figure $3 \mathbf{A}$ right and $\mathbf{G}$ right). An entity, identifiable by the interference fringes distinct from those arising from the ice surface, covered the surface of the ice and showed fluidic behaviour. This indicated the existence of a liquid that forms an interface with bulk water, even when the ice polymorph is not ice III. As in the case of ice III, the liquid layer changed its behaviour on compression or decompression. The thin liquid layer tended to expand on pressurization (Figure $3 \mathbf{B}-\mathbf{I}$ ) and to shrink on depressurization (Figure $3 \mathbf{A}$ and $\mathbf{B}$ ). This behaviour differs from that observed in previous experimental studies by light-scattering methods ${ }^{24}$, which found that the interfacial layer thickened to approximately $200 \mu \mathrm{m}$ as the growth rate of ice Ih decreased toward zero, suggesting that our liquid thin layer has different properties to those identified by previous studies. The visualization of a liquid layer on both ice III and ice VI suggests that the formation of a liquid layer distinguishable from bulk water is a universal phenomenon. 


\section{Density of the separated liquid layer.}

In relation to the structure of interfacial layer, it is important to know whether our newly discovered liquid distinct from bulk water is denser than bulk water. If we apply Young's equation, the relationship between the interfacial free energies is as follows:

$$
\gamma_{\mathrm{IW}}=\gamma_{\mathrm{IL}}+\gamma_{\mathrm{LW}} \cos \theta
$$

where $\gamma_{\mathrm{IW}}, \gamma_{\mathrm{IL}}$, and $\gamma_{\mathrm{LW}}$ are the interfacial free energies for ice/bulk water, ice/newly found liquid, and newly found liquid/bulk water, respectively, and $\theta$ is the wetting angle at the interfaces between ice and the newly discovered liquid and between bulk water and the newly discovered liquid. For ice VI, the wetting angle must be less than $90^{\circ}$, otherwise the liquid layer becomes improbably thick $(<50 \mu \mathrm{m})$. This is because the thickness of a wetted droplet $(t)$ can be expressed as follows:

$$
t=r(1-\cos \theta)
$$

when a spherical droplet with radius $r$ wets a substrate. When the spherical droplet wets a substrate with the condition $90^{\circ}<\theta<180^{\circ}$, Equation 2 gives $r<t<2 r$. Because $2 r$ corresponds to the inplane size of the liquid layer, and the size can be 100-200 $\mu \mathrm{m}$ (Figure 3 B and F), the thickness must be at least $50-100 \mu \mathrm{m}$ if the wetting angle is assumed to be more than $90^{\circ}$. The constraint that $\theta$ is less than $90^{\circ}$ leads to $\gamma_{\mathrm{IW}}>\gamma_{\mathrm{IL}}$, implying that the structure of the thin liquid layer is similar to that of ice VI, which has a higher density than that of bulk water.

The thickness of the liquid layer covering the surface of ice III was estimated to be approximately $2 \mu \mathrm{m}$ by measuring the wavelength of the adhesion-induced instability pattern ${ }^{26}$ that formed when 
the thin liquid layer contacted the roof of the anvil cell (SI Text S1 and Figure S2-S4). The diameter of the observed droplets reached approximately $8 \mu \mathrm{m}$. This relationship between the thickness and diameter indicates that $\theta$ is less than $90^{\circ}$. Similarly, the structure of the droplets is more similar to that of ice III than to that of bulk water. A molecular-dynamics (MD) simulation based on the TIP4P/Ice model ${ }^{27}$ indicated that the liquid water, when compressed to conditions for the coexistence of ice III and water, exhibits a similar structure to the crystal structure of ice III, unlike the structures observed under other conditions of pressure (SI Text S2 and Figure S5). This suggests that liquid water with a similar structure to ice III can exist, although it is unclear that the structural change would lead to separation between the thin liquid layer and bulk water. Consequently, the densities of the thin liquid layers on ice III and ice VI are estimated to be higher than that of bulk water. Therefore, the thin liquid layer is henceforth referred to as the high-density liquid layer (HDLL)

\section{Bicontinuous pattern formation in the HDLL}

We examined further the relationship between the dynamics of the pattern of the HDLL on an ice III crystal and the state of compression or decompression. The ice surface exhibited a smooth and uniform morphology when the crystal was grown by compression (Figure 4 A $0 \mathrm{~s}-10 \mathrm{~s}$ ). However, this smooth surface morphology changed to a pattern with a periodic wave once compression was stopped (Figure 4 A 10 s-13 s and insets). A surface with a wavelike morphology then developed into a HDLL with concave gaps with a size of a few tens of micrometres. (Figure 4 A 19 s). Further compression after the development of the HDLL with concave gaps caused the concave gaps to disappear through two-dimensional growth of the HDLL (Figure 4 A 19 s-25s). Eventually, the HDLL recovered to its initial smooth and uniform morphology on further 
compression (Figure 4 A 25 s). This indicates that the initial smooth surface was actually covered by the HDLL, although the initial surface morphology apparently consisted of an interface between the faceted ice and bulk water. When compression was stopped (Figure 4 B $0 \mathrm{~s}-11 \mathrm{~s}$ ), the smooth HDLL changed to a bicontinuous pattern via a wavelike pattern, as typically observed in spinodal decomposition of immiscible liquids in a two-component system ${ }^{28}$. This bicontinuous pattern also suggests the existence of two kinds of immiscible liquid at the interface between the ice and bulk water.

\section{CONCLUSION AND OUTLOOK}

In situ optical microscopic observation revealed that a macroscopic liquid layer denser than bulk water formed on the surfaces of ice III and VI as a water-ice interfacial layer when these ices were grown or melted by pressurization or depressurization. The HDLL was found to be separated from bulk water by a clear interface, and it showed a bicontinuous pattern at the water-ice interface. This indicates that two kinds of structured water immiscible with each other appeared at the waterice interface. Our observations suggest a novel physical picture of the water-ice interface that differs from the classical one. Whereas in the conventional picture, water, before its incorporation into bulk ice, continuously changes its structure, toward that of the ice, in an interfacial layer a few nanometres thick at most, we found that the bulk water, before its incorporation into ice, discretely changed its structure to that of a HDLL that was more compatible with the structure of the ice and was a few micrometres thick. This finding may redefine conventional thought regarding the waterice interface, leading to revisiting of chemical reactions catalysed by this interface.

The revealed physical picture of the interface is analogous to the actively debated picture of nonclassical crystal growth ${ }^{29,30}$, which deviates from assumptions in classical crystal-growth 
theory, for example, in respect of building units of a crystal incorporating into the crystal without any structural changes. In nonclassical growth, the building units in an ambient phase may adopt a conformation that is kinetically favourable and thermodynamically close to the crystalline phase before their incorporation into crystals. The corresponding conformation is often a fluid phase with higher density than the ambient phase ${ }^{31-33}$. This might imply that the conformation of the water molecular clusters comprising the HDLL have an underlying conformation as a metastable phase like the "high-density liquid" and "low-density liquid" postulated in the second-critical-point hypothesis that accounts for the anomalous properties of water ${ }^{34-36}$.

Moreover, because ice III has a chiral crystal structure ${ }^{37}$, further studies should provide insights into chiral separation in a theoretically predicted supercooled water composed of a racemic mixture of ordered chiral clusters ${ }^{38}$ in the context of biohomochirality. The new physical picture

of the interface will stimulate developments in relation to crystal growth in the synthesis of functional materials from melts, the nature of the interiors of small bodies in the Solar System, meteorite impacts, and so on. Our unexpected findings offer novel a platform, the water-ice interface, for opening a range of novel scientific fields.

\section{METHODS}

In situ polarized-light microscopic observation of an ice III crystal grown/melted in liquid water in a sapphire anvil cell

Ultrapure water from an ultrapure-water-producing apparatus (Simplicity UV; Merck Millipore, Burlington, MA, USA) fed with distilled water (Kyoei Seiyaku Co., Tokyo) was used. Ice III was 
crystallized by compressing the ultrapure water to the conditions for coexistence of ice III and liquid water ( $\left.248 \mathrm{MPa},-20^{\circ} \mathrm{C}\right)$ by using a symmetric-type sapphire anvil cell (SEED; Syntek Co. Ltd, Yokohama) in a low-temperature room kept at $-20^{\circ} \mathrm{C}$. Growth and melting of the ice III crystal were controlled by manual adjustment of the pressure-adjusting screw of the sapphire anvil cell. Compression and decompression resulted in growth and melting of the ice III crystal, respectively. The dynamics of crystallization/melting of liquid water in the cell were observed in situ by using an inverted polarized-light microscope (IX71; Olympus Corp., Tokyo) located in the low-temperature room. The microscopic images were recorded in situ by using a CCD camera (CN43H; Elmo Co. Ltd, Nagoya).

\section{In situ observations using an optical microscope with a Fizeau interferometer of an ice VI}

Ice VI was crystallized by compressing ultrapure water to the conditions for coexistence of ice VI and liquid water $\left(\sim 954 \mathrm{MPa}, 25^{\circ} \mathrm{C}\right)$ by using the sapphire anvil cell in a room at $25^{\circ} \mathrm{C}$. Growth and melting of the crystal were controlled by the same procedure written above. The dynamics of crystallization/melting of the crystal in water were observed in situ by using an inverted optical microscope (IX71; Olympus Corp., Tokyo) equipped with a Fizeau interferometer using a He-Ne laser (05-LHR-211; CVI Melles Griot, Albuquerque, NM; $\lambda=632.8 \mathrm{~nm}$ ) as the light source (SI Figure S1 A). The laser was introduced into a polarizer to adjust its intensity and direction of polarization. The beam was then focused by an objective lens (LMPlanFL N; Olympus) and passed to a polarization beam splitter (PBS) to introduce the laser into the optical path of the microscope by reflecting the laser in the vertical direction. The focused light reflected by the PBS was next introduced to an objective lens for sample observation, which collimated the introduced light. The ice crystal was irradiated by the collimated laser. The incident laser light and the light reflected by interfaces interfered with each other to produce interference fringes (Figure S1 B). The 
interference fringes were recorded in situ by a CCD camera (UI-3180CP-C-HQ Rev.2; IDS, Obersulm, Germany).

\section{AUTHOR INFORMATION}

\section{Corresponding Authors}

Hiromasa Niinomi - Institute for Materials Research, Tohoku University, Sendai, Miyagi, 9808577, Japan

E-mail: h.niinomi@imr.tohoku.ac.jp; niinomi37@gmail.com,

Telephone: $+81-22-215-2103$

Yuki Kimura - Institute of Low Temperature Science, Hokkaido University, Kita-19, Nishi-8,

Kita-ku, Sapporo, 060-0819, Japan

E-mail: ykimura@lowtem.hokudai.ac.jp

Telephone: +81-11-706-7666

\section{Authors}

Tomoya Yamazaki - Institute of Low Temperature Science, Hokkaido University, Kita-19,

Nishi-8, Kita-ku, Sapporo, 060-0819, Japan

Hiroki Nada - National Institute of Advanced Industrial Science and Technology (AIST), 16-1

Onogawa, Tsukuba 305-8569, Japan

Tetsuya Hama - Komaba Institute for Science, the University of Tokyo, 3-8-1 Komaba, Meguro, Tokyo 153-8902, Japan 
Akira Kouchi - Institute of Low Temperature Science, Hokkaido University, Kita-19, Nishi-8, Kita-ku, Sapporo, 060-0819, Japan

Junpei T. Okada - Institute for Materials Research, Tohoku University, Sendai, Miyagi, 9808577, Japan

Jun Nozawa - Institute for Materials Research, Tohoku University, Sendai, Miyagi, 980-8577, Japan

Satoshi Uda - Institute for Materials Research, Tohoku University, Sendai, Miyagi, 980-8577, Japan

\section{Notes}

The authors declare no competing financial interest.

\section{ASSOCIATED CONTENT}

\section{Supporting Information (SI).}

The Supporting Information is available free of charge on the ACS Publications website at XXXX.

SI Text S1. Estimation of the thickness of the thin liquid layer from the wavelength of adhesioninduced fingering instability, SI Text S2. Molecular dynamics (MD) simulations of liquid water, SI Figure S1. Optical setup of the microscope with a Fizeau interferometer, SI Figure S2. Adhesion-induced fingering instability of an elastic thin film sandwiched between two rigid surfaces, SI Figure S3. Fingering instability of the HDLL on an ice III crystal induced by adhesion of the HDLL to the roof of the anvil cell, SI Figure S4. Measurement of the wavelength of the adhesion-induced fingering instability of HDLL on a growing ice III crystal, SI Figure S5. 
Analysis of the liquid structure of the water under ice III-liquid two-phase coexistence conditions, based on MD numerical simulation, Legends for SI Movies S1-S4, SI References (PDF)

SI Movie S1. In situ observation of emergence of a thin liquid layer and droplets distinguishable from bulk water on a surface of an ice III crystal by polarized-light microscopy (MPEG), SI Movie S2. In situ observation of emergence of a thin liquid layer at the interface between ice VI and bulk water by optical microscope with a Fizeau interferometer (MPEG), SI Movie S3. In situ observation of change in the pattern of HDLL depending on the compression or release of compression, and bicontinuous pattern formation by an optical microscopy (MPEG), SI Movie S4. In situ observation of adhesion-induced fingering instability of HDLL on an ice III crystal by polarized-light microscopy (MPEG)

\section{ACKNOWLEDGMENT}

This research was supported by the Grant for Joint Research Program of the Institute of Low Temperature Science, Hokkaido University (18K001).

\section{REFERENCES}

1. Molina, M. J. The Probable Role of Stratospheric 'Ice' Clouds: Heterogeneous Chemistry of the 'Ozone Hole'. in The Chemistry of the Atmosphere: Its Impact on Global Change; Calvert, J. G., Ed.; Blackwell Scientific Publications, 1994.

2. Dash, J. G.; Rempel, A. W.; Wettlaufer, J. S. The physics of premelted ice and its geophysical consequences. Rev. Mod. Phys. 2006, 78, 695-741. 
3. Stibal, M.; Šabaká, M.; Žárský, J. Biological processes on glacier and ice sheet surfaces. Nat. Geosci. 2012, 5, 771-774.

4. Shiraiwa, M.; Ammann, M.; Koop, T.; Pöschl, U. Gas uptake and chemical aging of semisolid organic aerosol particles. Proc. Natl. Acad. Sci. U.S.A. 2011, 108, 11003-11008.

5. Nagata, Y.; Hama, T.; Backus, E. H. G.; Mezger, M.; Bonn, D.; Bonn, M.; Sazaki, G. The surface of ice under equilibrium and nonequilibrium conditions. Acc. Chem. Res. 2019, 52, $1006-1015$.

6. Kahan, T. F.; Wren, S. N.; Donaldson, D. J. A pinch of salt is all it takes: Chemistry at the frozen water surface. Acc. Chem. Res. 2014, 47, 1587-1594.

7. Faraday, M. Experimental Researches in Chemistry and Physics; Taylor and Francis, 1859.

8. Slater, B.; Michaelides, A. Surface premelting of water ice. Nat. Rev. Chem. 2019, 3, 172-188.

9. Tang, F.; Ohto, T.; Sun, S.; Rouxel, J. R.; Imoto, S.; Backus, E. H. G.; Mukamel, S.; Bonn, M.; Nagata, Y. Molecular structure and modeling of water-air and ice-air interfaces monitored by sum-frequency generation. Chem. Rev. 2010, 120, 3633-3667.

10. Sazaki, G.; Zepeda, S.; Nakatsubo, S.; Yokomine, M.; Furukawa, Y. Quasi-liquid layers on ice crystal surfaces are made up of two different phases. Proc. Natl. Acad. Sci. U.S.A. 2012, 109, $1052-1055$.

11. Murata, K.; Asakawa, H.; Nagashima, K.; Furukawa, Y.; Sazaki, G. Thermodynamic origin of surface melting on ice crystals. Proc. Natl. Acad. Sci. U.S.A. 2016, 113, E6741-E6748.

12. Nagashima, K.; Sazaki, G.; Hama, T.; Murata, K.; Furukawa, Y. Uptake mechanism of atmospheric hydrogen chloride gas in ice crystals via hydrochloric acid droplets. Cryst. Growth Des. 2018, 18, 4117-4122. 
13. Murata, K.; Asakawa, H.; Nagashima, K.; Furukawa, Y.; Sazaki, G. In situ determination of surface tension-to-shear viscosity ratio for quasiliquid layers on ice crystal surfaces. Phys. Rev. Lett. 2015, 115, 256103.

14. Canale, L.; Comtet, J.; Niguès, A.; Cohen, C.; Clanet, C.; Siria, A.; Bocquet, L. Nanorheology of interfacial water during ice gliding. Phys. Rev. X 2019, 9, 041025.

15. Dash, J. G. Surface melting. Contemp. Phys. 1989, 30, 89-100.

16. Russell, M. J.; Murray, A. E.; Hand, K. P. The possible emergence of life and differentiation of a shallow biosphere on irradiated icy worlds: The example of Europa. Astrobiology 2017, $17,1265-1273$.

17. Nada, H. Anisotropy in geometrically rough structure of ice prismatic plane interface during growth: Development of a modified six-site model of $\mathrm{H}_{2} \mathrm{O}$ and a molecular dynamics simulation. J. Chem. Phys. 2016, 145, 244706.

18. Wang, R.; Xu, L.-M.; Wang, F. Molecular-scale processes affecting growth rates of ice at moderate supercooling. Front. Phys. 2018, 13, 138116.

19. Gulam Razul, M. S.; Kusalik, P. G. Crystal growth investigations of ice/water interfaces from molecular dynamics simulations: Profile functions and average properties. J. Chem. Phys. 2011, $134,014710$.

20. Kim, Y.-J.; Lee, Y.-H.; Lee, S.; Nada, H.; Lee, G.-W. Shock growth of ice crystal near equilibrium melting pressure under dynamic compression. Proc. Natl. Acad. Sci. U.S.A. 2019, $116,8679-8684$.

21. Güttinger, H.; Bilgram, J. H.; Känzig, W. Dynamic light scattering at the ice water interface during freezing. J. Phys. Chem. Solids 1979, 40, 55-66. 
22. Brown, R. A.; Keizer, J.; Stelger, U.; Yeh, Y. Enhanced light scattering at the ice-water interface during freezing. J. Phys. Chem. 1983, 87, 4135-4138.

23. Bilgram, J. H. Dynamics at the solid-liquid transition: Experiments at the freezing point. Phys. Rep. 1987, 153, 1-89.

24. Halter, P. U.; Bilgram, J. H.; Känzig, W. Properties of the solid-liquid interface layer of growing ice crystals: A Raman and Rayleigh scattering study. J. Chem. Phys. 1988, 89, 26222629.

25. Lobban, C.; Finney, J. L.; Kuhs, W. F. The structure of a new phase of ice. Nature 1998, 391, $268-270$.

26. Chaudhury, M. K.; Chakrabarti, A.; Ghatak, A. Adhesion-induced instabilities and pattern formation in thin films of elastomers and gels. Eur. Phys. J. E: Soft Matter Biol. Phys. 2015, $38,82$.

27. Abascal, J. L. F.; San, E.; Fernádez, R. G.; Bega, C. A potential model for the study of ices and amorphous water: TIP4P/Ice. J. Chem. Phys. 2005, 122, 234511.

28. Lopez-Barron, C. R.; Macosko, C. W. Characterizing interface shape evolution in immiscible polymer blends via 3D image analysis. Langmuir 2009, 25, 9392-9404.

29. Jehannin, M.; Rao, A.; Cölfen, H. New horizons of nonclassical crystallization. J. Am. Chem. Soc. 2019, 141, 10120-10136.

30. Nada, H. Pathways for the formation of ice polymorphs from water predicted by a metadynamics method. Sci. Rep. 2020, 10, 4708.

31. ten Wolde, P. R.; Frenkel, D. Enhancement of Protein Crystal Nucleation by Critical Density Fluctuations. Science 1997, 277, 1975-1977. 
32. Wedekind, J.; Xu, L.; Buldyrev, S. V.; Stanley, H. E.; Reguera, D.; Franzese, G. Optimization of crystal nucleation close to a metastable fluid-fluid phase transition. Sci. Rep. 2015, 5, 11260.

33. Desgranges, C.; Delhommelle, J. Free energy calculations along entropic pathways. I. Homogeneous vapor-liquid nucleation for atomic and molecular systems. J. Chem. Phys. 2016, $145,204112$.

34. Martelli, F.; Crain, J.; Franzese, G. Network Topology in Water Nanoconfined between Phospholipid Membranes. ACS Nano 2020, in press, DOI:10.1021/acsnano.0c02984.

35. Poole, P. H.; Sciortino, F.; Essmann, U.; Stanley, H. E. Phase behaviour of metastable water. Nature 1992, 360, 324-328.

36. Nilsson, A.; Pettersson, L. G. M. The structural origin of anomalous properties of liquid water. Nat. Commun. 2015, 6, 8998.

37. Kamb, B.; Prakash A. Structure of Ice III. Acta Crystallogr., Sect. 1968, B24, 1317-1327.

38. Matsumoto, M.; Yagasaki, T.; Tanaka H. Chiral ordering in supercooled liquid water and amorphous ice. Phys. Rev. Lett. 2015, 115, 197801. 


\section{FIGURES}

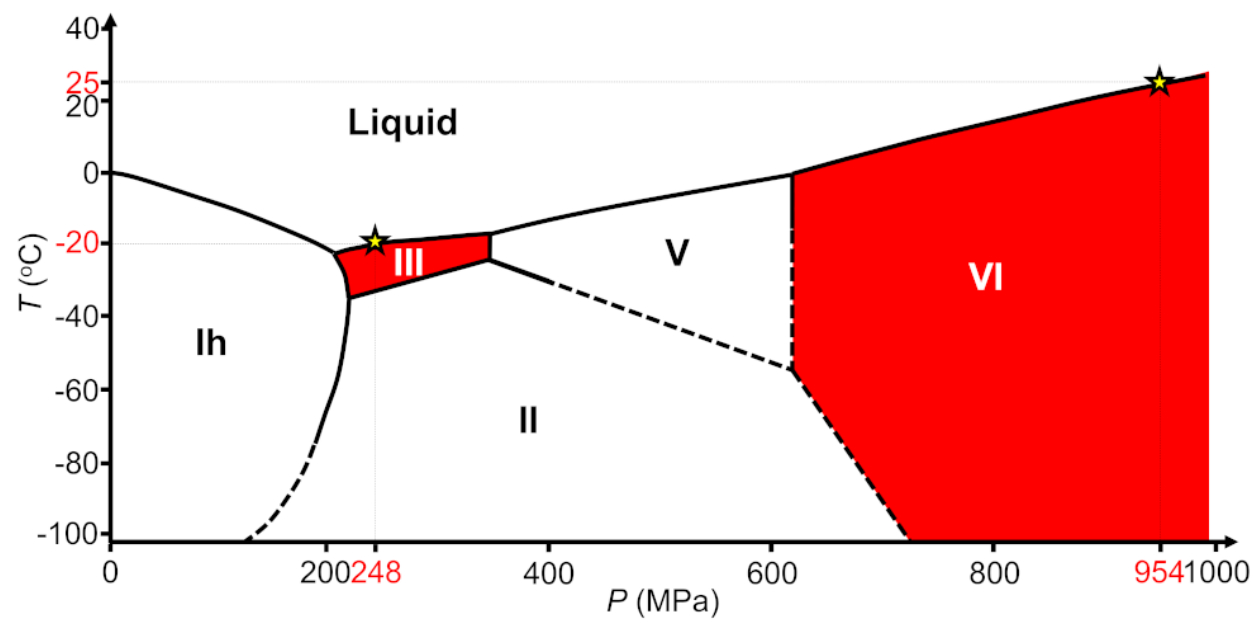

Figure 1. Experimental conditions plotted on the phase diagram of water ${ }^{25}$. Phase diagram of water and our experimental conditions for the observations on ice III and VI. The two red-coloured regions indicate the stable regions for ice III and VI, respectively. Our observations were performed at the liquid-ice coexistence conditions shown by stars, at $-20{ }^{\circ} \mathrm{C}$ and $248 \mathrm{MPa}$ for ice III, and at $25^{\circ} \mathrm{C}$ and $954 \mathrm{MPa}$ for ice VI. 

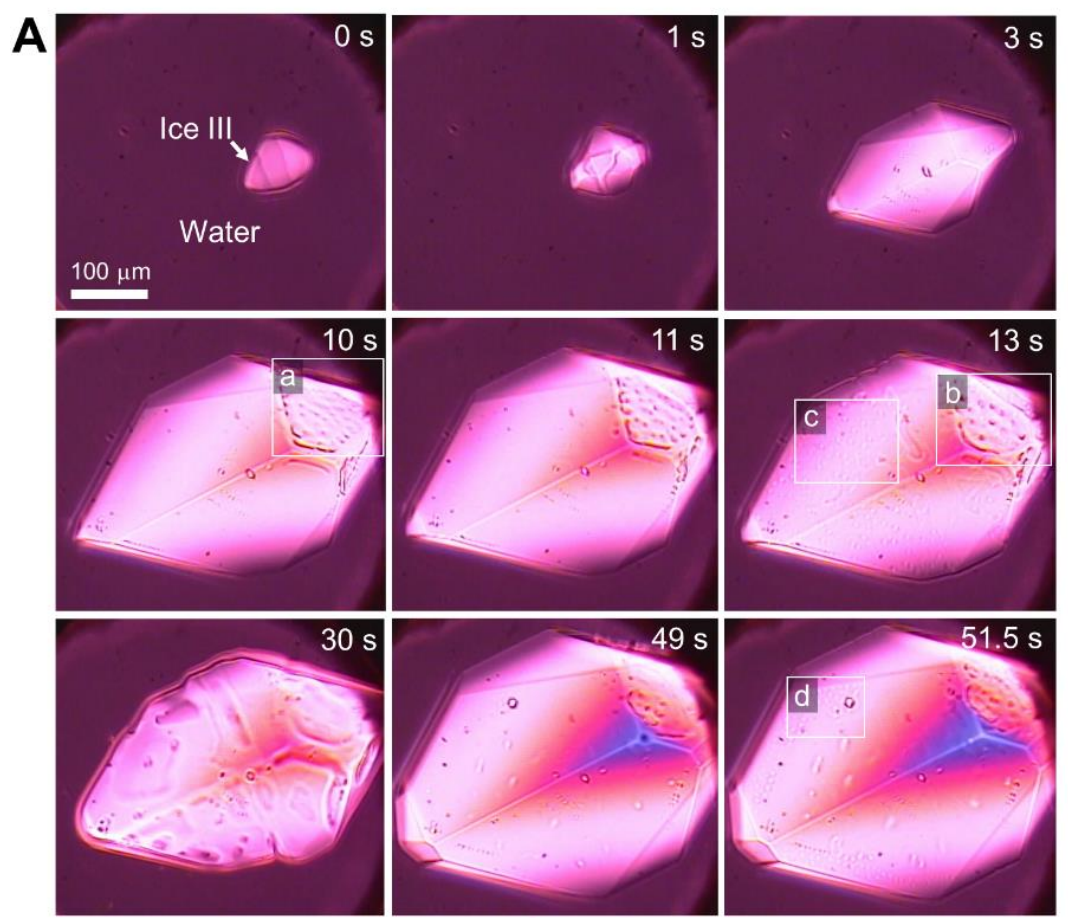

$0 s \Rightarrow 1 s \rightarrow 3 s \rightarrow 10 s \rightarrow 11 s \Rightarrow 13 s \rightarrow 30 s \rightarrow 49 s \rightarrow 51.5 s$

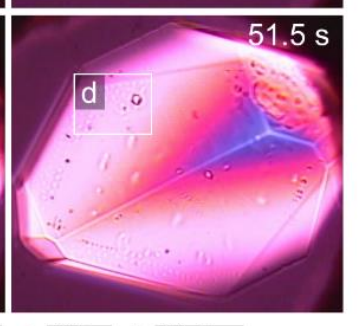

Release
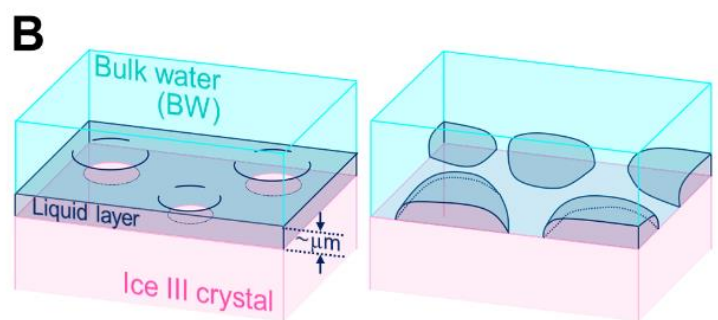

C
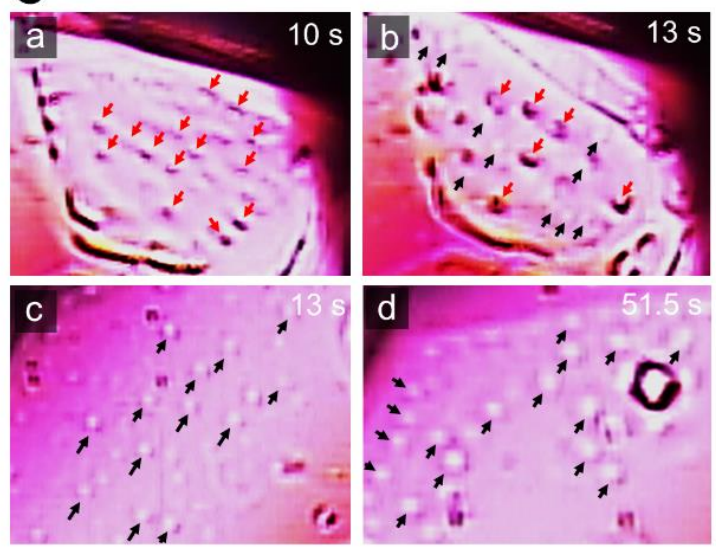

Figure 2. Emergence of a thin liquid layer and droplets distinguishable from bulk water on the surface of an ice III crystal. (A) Polarized-light photomicrographs of an ice III crystal grown/melted in water by compression/decompression in a sapphire anvil cell at $-20{ }^{\circ} \mathrm{C}$ (SI Movie S1). The magenta, cyan, and grey arrows at the bottom indicate the operations of compression, decompression, and release, respectively. (B) Schematic representations of two kinds of typical patterns of the newly found liquid: the left is a thin layer with concave holes, and the right consists of droplets. (C) Magnified images of rectangles a-d in micrographs A $10 \mathrm{~s}, 13 \mathrm{~s}$ and $51.5 \mathrm{~s}$, respectively. The red and black arrows in images a-d indicate concave holes in the liquid layer $(\mathbf{B}$, left) and liquid droplets (B, right), respectively. The coexistence of liquid water and ice III indicates that the pressure was approximately $248 \mathrm{MPa}$, in accordance with the phase diagram of water (Figure 1). The scale bar is $100 \mu \mathrm{m}$. 

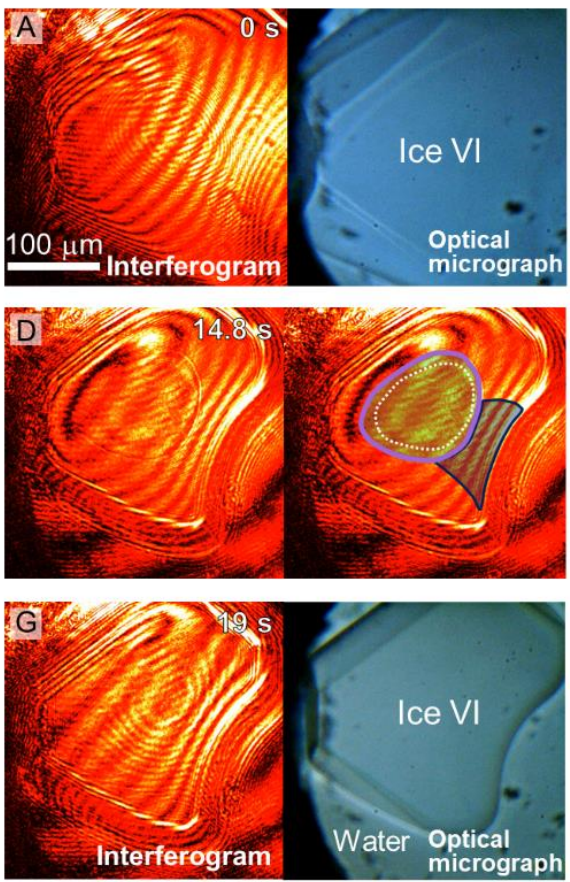

$\mathrm{A} \Rightarrow \mathrm{B} \Rightarrow \mathrm{C} \Rightarrow \mathrm{D} \Rightarrow \mathrm{E} \Rightarrow \mathrm{F}$
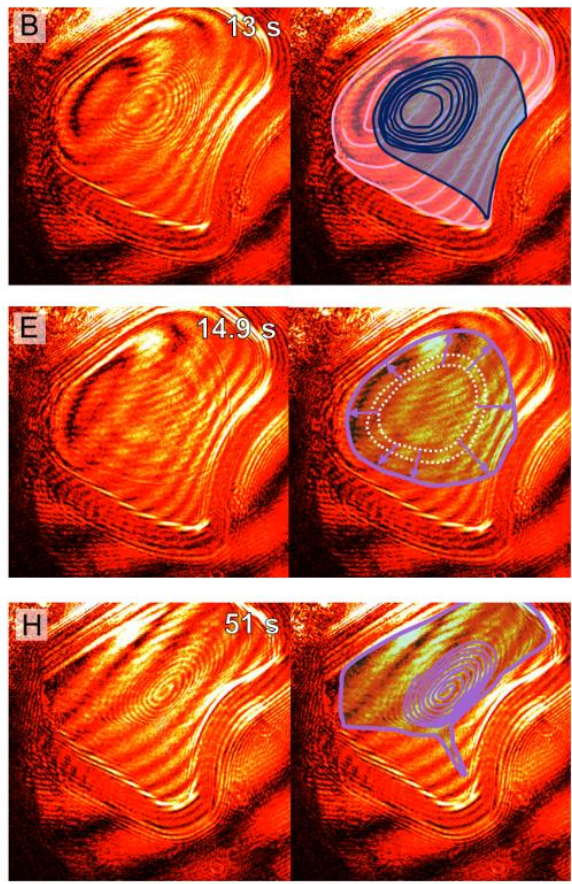

$\mathrm{F} \rightarrow \mathrm{G} \underset{\text { Release }}{\rightarrow} \rightarrow \mathrm{H}$
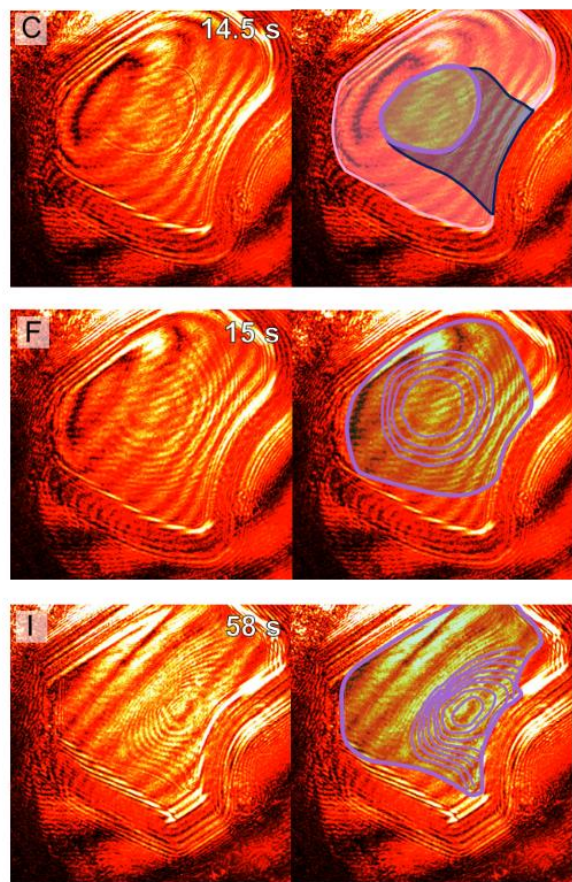

Figure 3. Emergence of a thin liquid layer at the interface between a growing or melting ice VI crystal and bulk water. (A)-(I), Photographs obtained by in situ observation with an optical microscope equipped with a Fizeau interferometer of an ice VI crystal in an anvil cell at $25^{\circ} \mathrm{C}$ (SI Movie S2). Each consists of two images. All the left-hand images show Fizeau interferograms of the microscopic view. The right-hand images in $\mathbf{A}$ and $\mathbf{G}$ are bright-field images without the interferometer. The other right-hand images in $\mathbf{B}-\mathbf{F}, \mathbf{H}$ and $\mathbf{I}$ are interferograms with eye guides to permit differentiation of the interference fringes from the surface of the thin liquid layer from those arising from the ice surface. The bright-magenta highlights and lines indicate the crystal surface and the interference fringes that originate from it, respectively. The dark-blue highlights and lines indicate the interference fringes from the surface of the thin liquid layer that initially formed, and the region covered by it. The region outlined by the solid purple line was covered by the thin liquid layer formed after compression. The dotted white line indicates the region covered by the layer in the previous photograph. The purple lines inside the region covered by the thin liquid layer indicate interference fringes originating from the surface of the thin layer formed after compression. The combination of coloured arrows A to I indicate the same information as the corresponding arrows in Figure 2. The coexistence of liquid water and ice VI indicates that the pressure was approximately $954 \mathrm{MPa}$ (Fig. 1). The scale bar is $100 \mu \mathrm{m}$. 

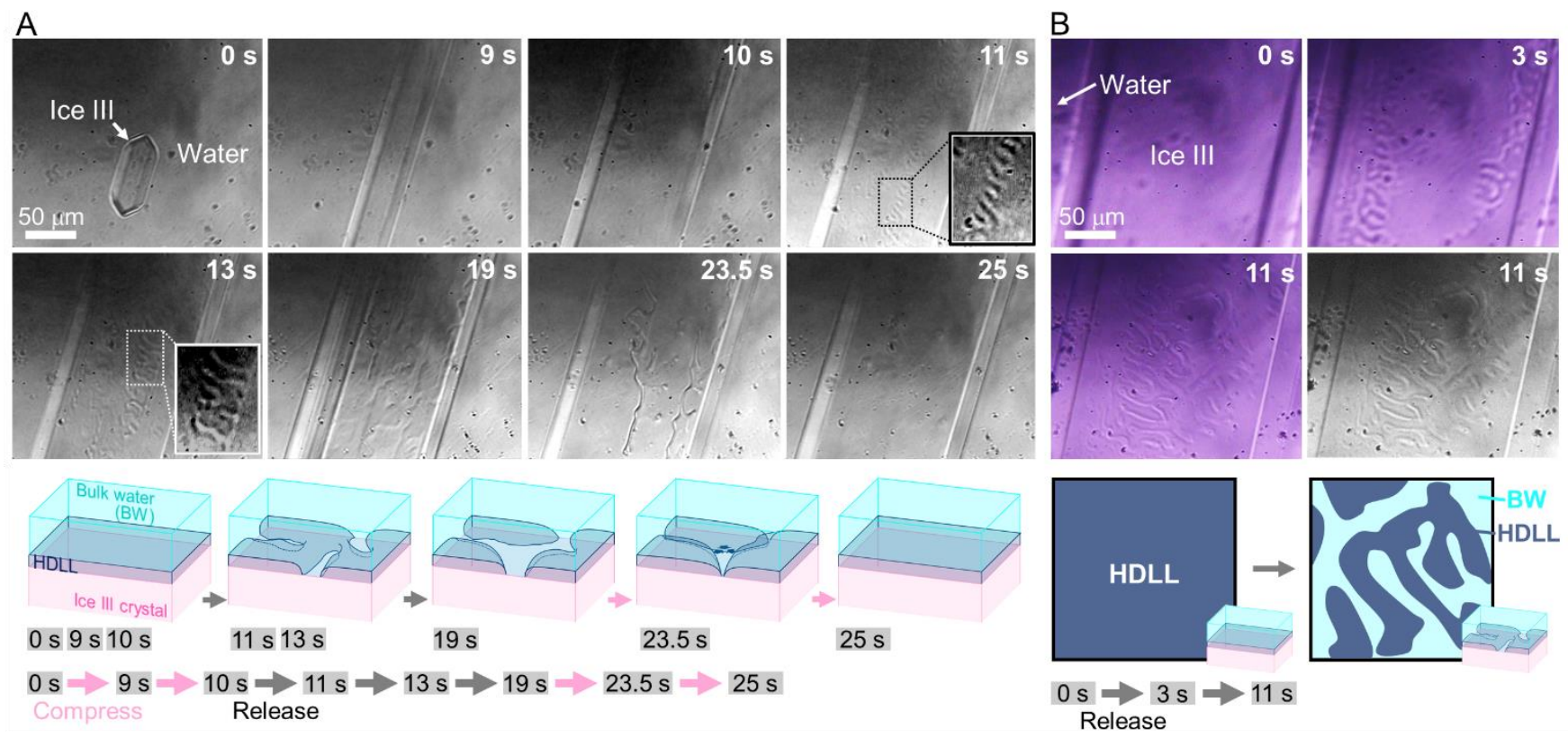

Figure 4. Changes in the pattern of the HDLL depending on compression or release of compression in the anvil cell. These images were recorded by in situ polarized-light microscopy of a crystal of ice III grown in the anvil cell at $-20^{\circ} \mathrm{C}$ (SI Movie S3). (A) The formation of periodic wavelike surface pattern on stopping the compression and the disappearance of the holes in the HDLL on compression. These are monochromatic micrographs from the in situ observations. The insets in the images $11 \mathrm{~s}$ and $13 \mathrm{~s}$ are magnified images of the regions outlined by the dotted black and white line, respectively. The schematic illustrations at the bottom show simplified patterns of the HDLL for each micrograph along with corresponding time. (B) The formation of a bicontinuous pattern of the HDLL on stopping compression. B $0 \mathrm{~s}-11 \mathrm{~s}$ (left) are micrographs from in situ observations. The left image for $11 \mathrm{~s}$ is a monochromatic form of the right image for $11 \mathrm{~s}$ to enhance the visibility of the bicontinuous pattern. The schematic illustrations at the bottom show the simplified pattern change of HDLL on release of compression. The combination of coloured arrows and times indicates the same information as the corresponding diagram in Figure 2. The scale bar is $50 \mu \mathrm{m}$. 


\section{Supporting Information for}

\section{High-Density Liquid Water at a Water-Ice Interface}

Hiromasa Niinomi ${ }^{* \dagger}$, Tomoya Yamazaki ${ }^{\ddagger}$, Hiroki Nada ${ }^{\S}$, Tetsuya Hama ${ }^{\mathbb{I}}$, Akira Kouchi ${ }^{\ddagger}$, Junpei T. Okada ${ }^{\dagger}$, Jun Nozawa ${ }^{\dagger}$, Satoshi Uda ${ }^{\dagger}$, and Yuki Kimura ${ }^{* \ddagger}$

${ }^{\dagger}$ Institute for Materials Research, Tohoku University, Sendai, Miyagi, 980-8577, Japan

${ }^{\ddagger}$ Institute of Low Temperature Science, Hokkaido University, Kita-19, Nishi-8, Kita-ku, Sapporo, 060-0819, Japan

${ }^{\S}$ National Institute of Advanced Industrial Science and Technology (AIST), 16-1

Onogawa, Tsukuba 305-8569, Japan

"Komaba Institute for Science, the University of Tokyo, 3-8-1 Komaba, Meguro, Tokyo 153-8902, Japan

Corresponding authors: Hiromasa Niinomi (HN), Yuki Kimura (YK)

Email: h.niinomi@imr.tohoku.ac.jp (HN),ykimura@lowtem.hokudai.ac.jp (YK)

\section{This PDF file includes:}

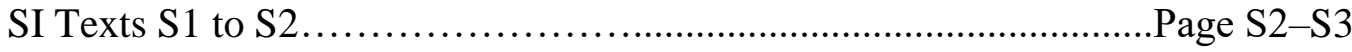

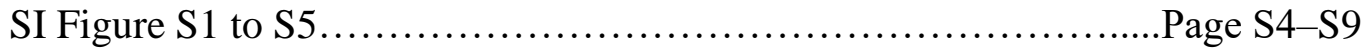

Legends for SI Movies S1 to S4 ...................................Page S10

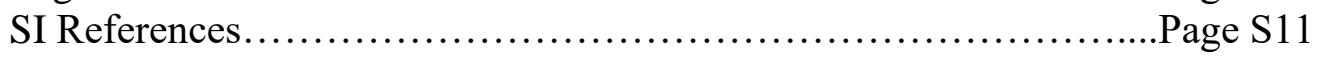

\section{Other supporting materials for this manuscript include the following:}

SI Movies S1 to S4 


\section{Supporting Information Text S1}

\section{Estimation of the thickness of the thin liquid layer from the wavelength of adhesion-}

induced fingering instability.

Adhesion-induced fingering instability is a phenomenon in which an elastic thin film exhibits instability by displaying fingering and labyrinth patterns when it is sandwiched between two rigid surfaces (Figure S2). When the distance between the two rigid surface is constant, the spacing of the intervals between successive branches of the fingering pattern is also constant. The periodicity in the spacing of each branch is the wavelength of the adhesion-induced fingering instability. Accumulated data from previous studies on this phenomenon suggest that the wavelength is solely determined by the distance between the two rigid surface and is independent of the physical properties of the elastic thin film, the surrounding medium, or the rigid surfaces ${ }^{1}$. The wavelength of the fingering instability $\lambda_{\mathrm{i}}$ and the distance between the two rigid surfaces $h$ are related according to the expression $\lambda_{\mathrm{i}}=3.8 h^{1}$. This relationship allows us to estimate the thickness of an elastic thin film interleaved between two rigid surfaces by measuring the wavelength of the adhesion-induced fingering instability.

In our experiments, the HDLL, the surface of ice crystal, and the roof of the sapphire anvil cell can be regarded, respectively, as an elastic thin film, a rigid surface, and a second rigid surface. Adhesion-induced fingering instability of the HDLL on the ice crystal appeared when the growing ice surface approached the roof of the anvil cell (Figure S3). Actually, the pattern of the fingering instability showed up just before the grown ice III crystal occupied the whole volume of the anvil cell (Figure S3 B), because at that stage the ice crystal surface supporting the thin liquid layer was sufficiently close to the roof of the anvil cell to cause an instability. We measured $\lambda_{\mathrm{i}}$ just as the instability pattern began to appear when the ice III crystal was grown by continuous compression (Figure S4). We did this because had we measured $\lambda_{\mathrm{i}}$ when the gap distance between the ice crystal surface and the roof of the anvil cell was less than the intrinsic thickness of the HDLL before adhesion to the roof, we would only have measured the gap distance, not the intrinsic thickness of the HDLL. The intrinsic thickness of the HDLL covering the growing ice III crystal surface was estimated to be approximately $2 \mu \mathrm{m}$ by substituting the measured $\lambda_{\mathrm{i}}$ value into the relationship $\lambda_{\mathrm{i}}=3.8 \mathrm{~h}$. This measurement showed that a HDLL with a thickness of about 2 $\mu \mathrm{m}$ covered the growing ice III crystal. 


\section{Supporting Information Text S2}

\section{Molecular dynamics (MD) simulations of liquid water.}

MD simulations of liquid water were performed by using a $2880 \mathrm{H}_{2} \mathrm{O}$ rectangularparallelepiped system onto which three-dimensional periodic boundary conditions were imposed. The TIP4P/Ice model ${ }^{3}$ was used to estimate intermolecular interactions, because this model produces a temperature-pressure range in which ice III exists stably. According to a report in the literature ${ }^{3}$, the ice III-water coexistence line occurs at a much lower temperature in the TIP4P/Ice model than in real systems. Consequently, the simulations were performed at $-39.2^{\circ} \mathrm{C}$, which corresponded to a simulation temperature of $-40.2^{\circ} \mathrm{C}$, because the melting point of ice Ih at $0.1 \mathrm{MPa}$ in the TIP4P/Ice model was $-0.95^{\circ} \mathrm{C}^{3}$, instead of the experimental temperature of $-20^{\circ} \mathrm{C}$. The pressure of ice III in equilibrium with water at $-39.2^{\circ} \mathrm{C}$ in the TIP4P/Ice model is approximately $310 \mathrm{MPa}^{3}$. We therefore performed our simulations at pressures of $1 \mathrm{~atm}, 150 \mathrm{MPa}, 310 \mathrm{MPa}, 450 \mathrm{~Pa}$, and $600 \mathrm{MPa}$ (Figure S5).

The computations were carried out by using a leap-frog algorithm with a time step of $2 \mathrm{fs}^{4}$. The temperature was maintained at $-39.2{ }^{\circ} \mathrm{C}$, and the pressure was set to the each of the values mentioned above by means of the method proposed by Berendsen and coworkers ${ }^{5}$. The thermal and pressure bath constants were set to 0.1 and $2.0 \mathrm{ps}$, respectively. The simulations were performed by using the MD software DL_POLY $2.20^{6}$. The Coulombic interaction was estimated by using the Ewald method with an accuracy of $10^{-6}$. The real-space cut-off distance was set to $1.0 \mathrm{~nm}$, and the Ewald convergence parameter was $3.208 \mathrm{~nm}^{-1}$. The Lennard-Jones interaction between the oxygen atoms of a pair of water molecules was truncated at an intermolecular distance of $1.0 \mathrm{~nm}$. The total run time was $50 \mathrm{~ns}$. The data for the first $40 \mathrm{~ns}$ at the beginning of the simulation were discarded as representing an equilibrium period, and the data for the subsequent $10 \mathrm{~ns}$ were used for the analysis of the water structure. 

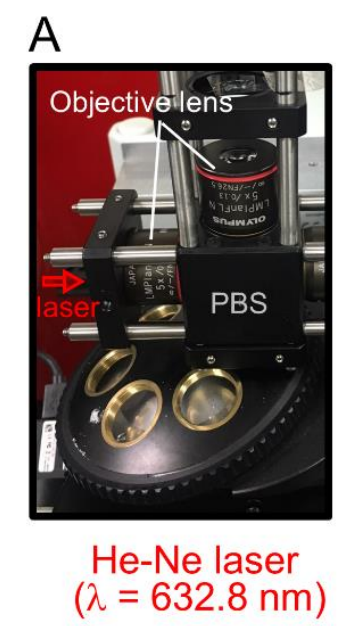

Sapphire anvil cell

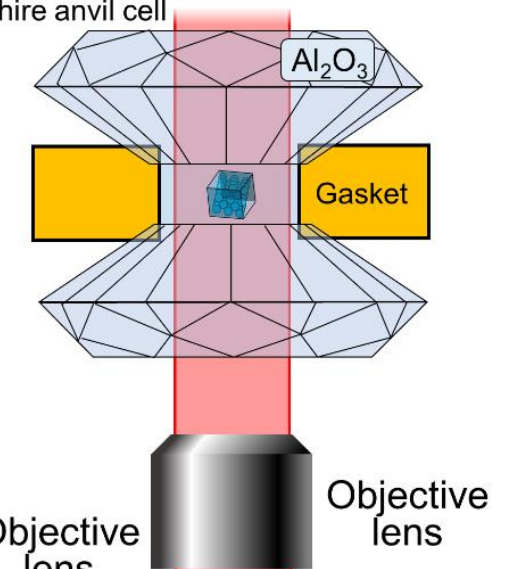

B
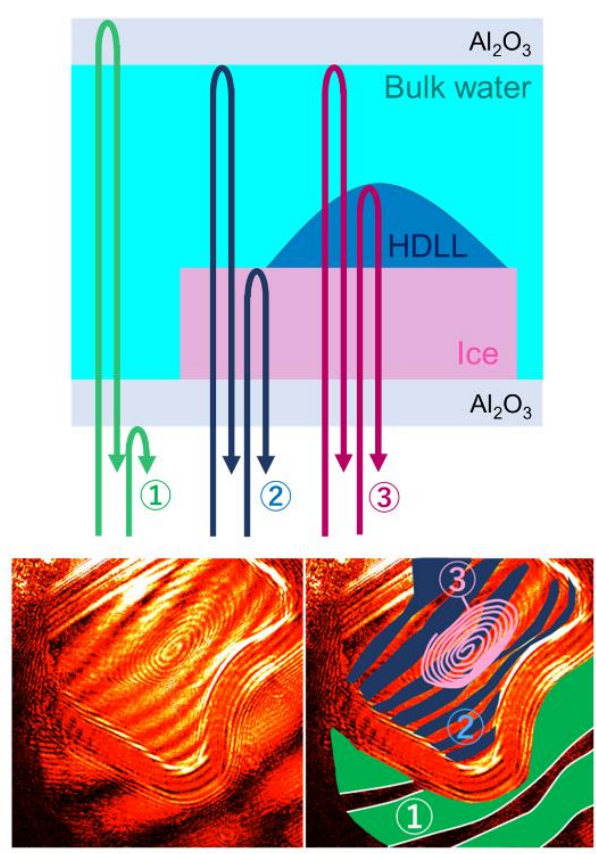

Figure. S1. Optical setup of the microscope with a Fizeau interferometer. (A) Schematic illustration showing the optical setup for the inverted optical microscope equipped with a Fizeau interferometer. The inset is a photograph showing the optical system mounted on the inverted optical microscope. (B) The upper schematic shows the conceivable optical paths that give rise to the three kinds of interference fringes seen during our in situ observations: (1) interference between light reflected by the air-sapphire anvil cell interfaces at the bottom and the top (green); (2) interference between the light reflected by the upper bulk water/sapphire interface and that reflected by the ice crystal/bulk water interface (dark blue); and (3) interference between light reflected by the upper bulk water/sapphire interface and that reflected by the HDLL/bulk water interface (purple). The lower images are examples that show each of the three kinds of interference fringe (left) and a schematic showing the correspondence between the fringes and the three conceivable interference optical paths (right). PBS = polarization beam splitter. 
A
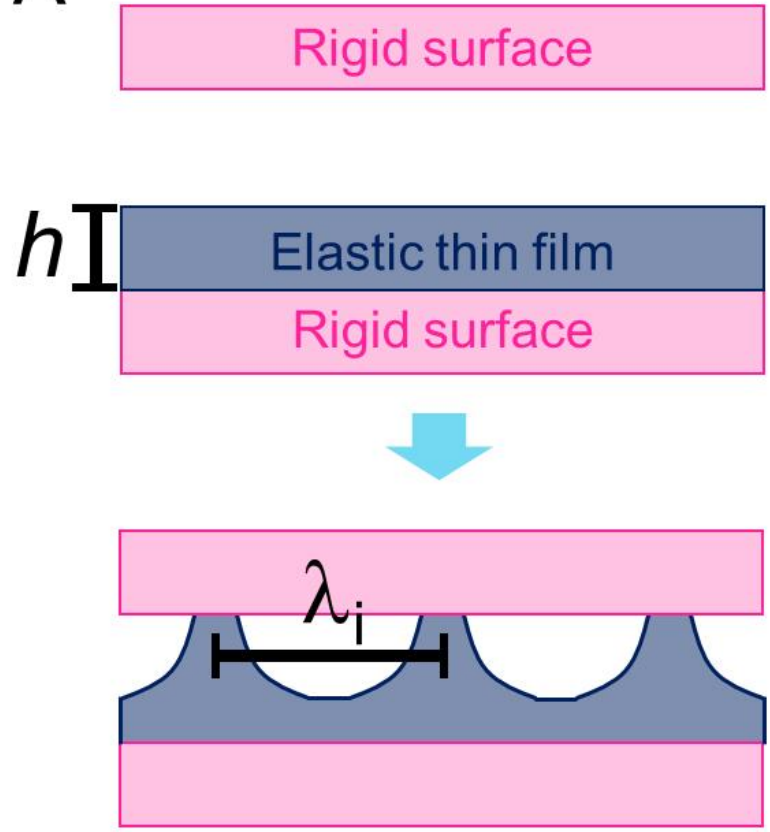

B

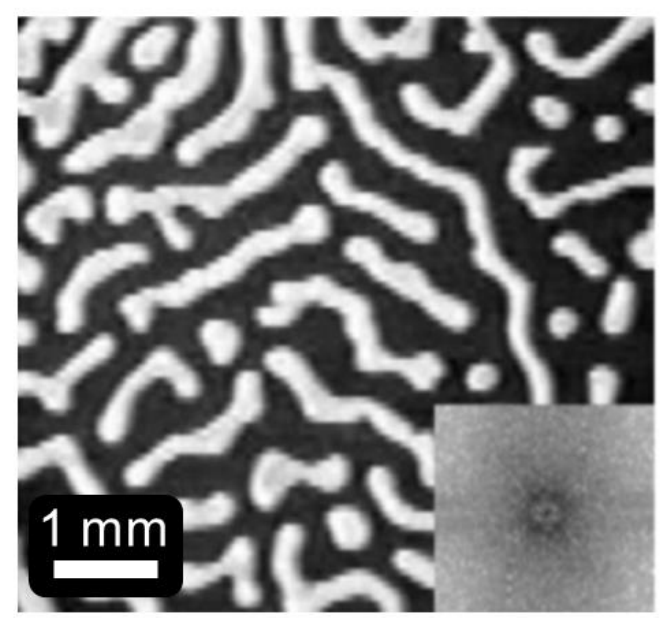

Figure S2. Adhesion-induced fingering instability of an elastic thin film sandwiched between two rigid surfaces. (A) Schematic representation of adhesion-induced fingering instability: $h$ is the thickness of the elastic thin film and $\lambda_{i}$ is the wavelength of the instability. (B) This is an example of a microscopic image of an adhesion-induced fingering instability pattern, obtained in a previous study. The inset shows the Fouriertransformed image of the microscopic view. The microscopic image is reprinted from Ref. 2 with the permission of the Taylor and Frances Group ${ }^{2}$. 


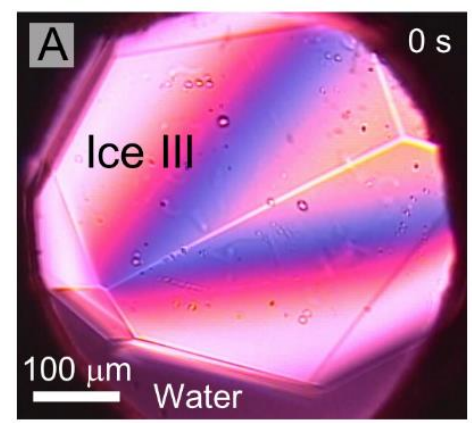

$A^{\prime}$

The roof of the anvil cell
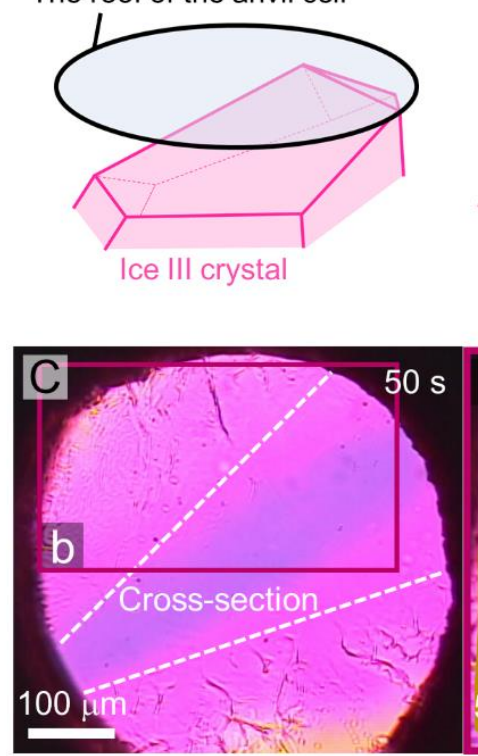

C'

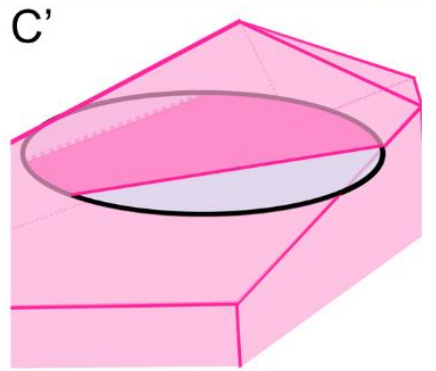

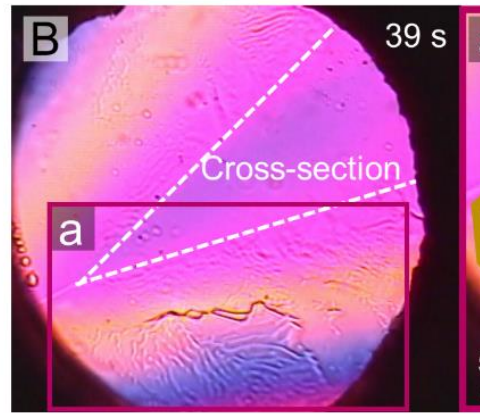

B'

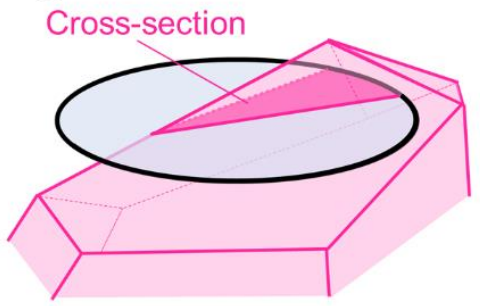

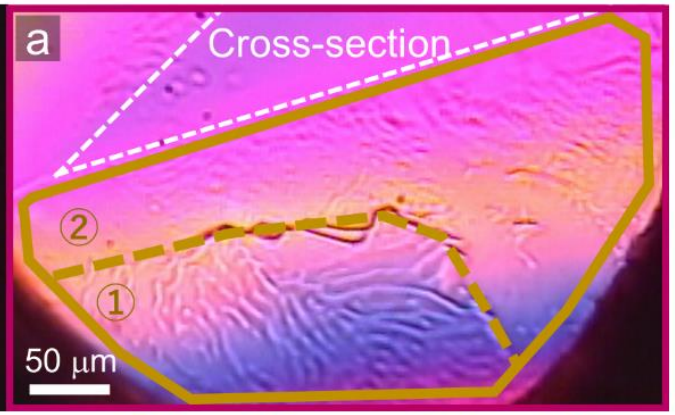

(1) $\lambda_{\mathrm{i}}=8.3 \mu \mathrm{m} \quad$ (2) $\lambda_{\mathrm{i}}=6.3 \mu \mathrm{m}$

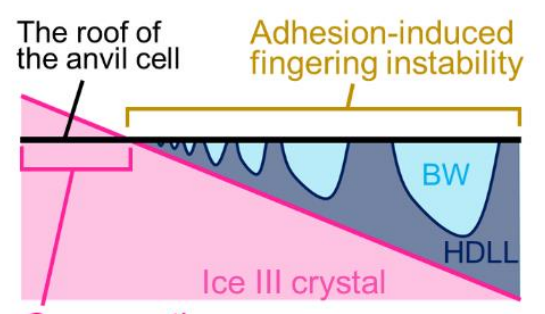

Cross-section
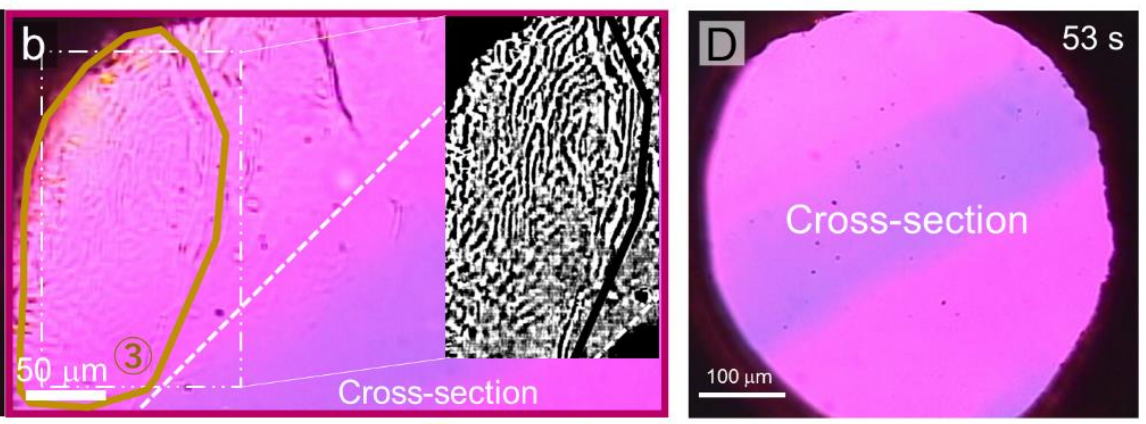

Adhesion-induced

fingering instability
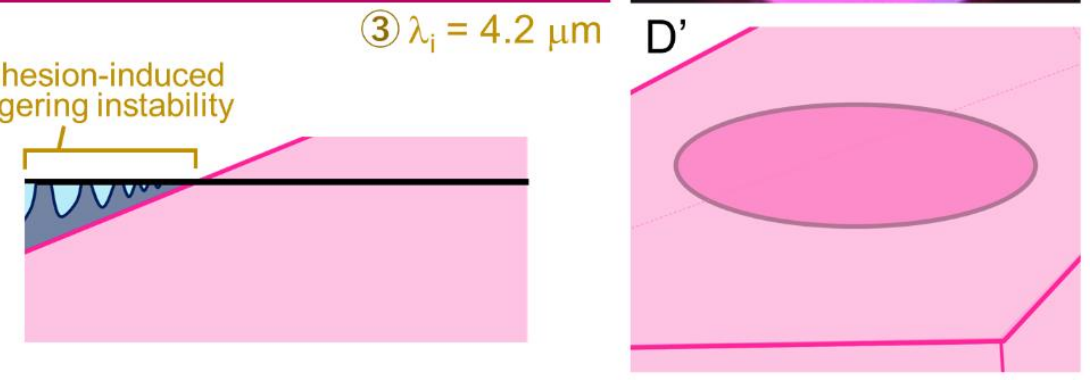

(3) $\lambda_{i}=4.2 \mu \mathrm{m}$

Figure S3. Fingering instability of the HDLL on an ice III crystal induced by adhesion of the HDLL to the roof of the anvil cell. (A)-(D) In situ polarized-light micrographs showing the emergence of the fingering instability induced by adhesion of the HDLL to the roof of the cell. (A')-(D') Corresponding schematic illustrations showing the geometrical relationship between the roof of the cell and the growing ice crystal in water. When the vertex of the crystal reached the roof of the cell through compression-induced crystal growth, an instability pattern appeared near the region where the crystal contacted the roof (A and $\mathbf{B})$. (A) The fingering instability cannot be seen, because the vertex of the crystal had not reached the roof of the anvil cell at this time. (B) The vertex of the crystal has reached the roof of the cell. The region outlined by the dotted line indicates the region where the grown crystal contacted the roof of the cell. The rectangle a indicates the region 
where a fingering instability pattern appeared as a result of the presence of a gap that permitted the HDLL to exist. A magnified view of region a is shown on the right-hand side. The region outlined by the solid ochre line indicates the region where the fingering instability pattern appeared. This region can be divided into two kinds of region, depending on the wavelength of the instability pattern. By taking the average of ten arbitrary measurements for each region, the wavelength in the region designated 1 was calculated to be $8.3 \mu \mathrm{m}$, whereas that in the region 2 was calculated to be $6.3 \mu \mathrm{m}$. The wavelength increased with increasing distance from the region where the crystal contacted the roof of the cell. The right-hand schematic in B' shows the geometrical relationship from the crosssectional view for the region delineated by the ochre line in region a. Because the gap between the crystal surface and the roof of the anvil cell increased with increasing horizontal distance from the region where the crystal surface contacts the roof of the anvil cell, it follows that the wavelength of the instability increased with distance from this region in accordance with the nature of the adhesion-induced fingering instabilities. (C) Further growth expanded the region of contact between the ice surface and the roof. A magnified view of region $b$ is shown on the right-hand side. Region 3 outlined by an ochre line, delineates where the instability pattern can be seen. The inset monochrome image corresponds to the region outlined by the line of alternating dots and dashes. The wavelength of the instability is measured to be $4.2 \mu \mathrm{m}$, which is shorter than that in micrograph $\mathbf{B}$. This is because the gap between the crystal surface and the roof of the anvil cell at this moment was smaller than that for region a as a result of crystal growth, as shown in the right-hand schematic $\mathbf{C}^{\prime}$. (D) The grown crystal occupied the entire volume of the cell. In agreement with the physical picture of adhesion-induced fingering instability, the pattern disappeared when the crystal contacted the whole area of the roof of the cell by filling the entire volume of the anvil cell. 

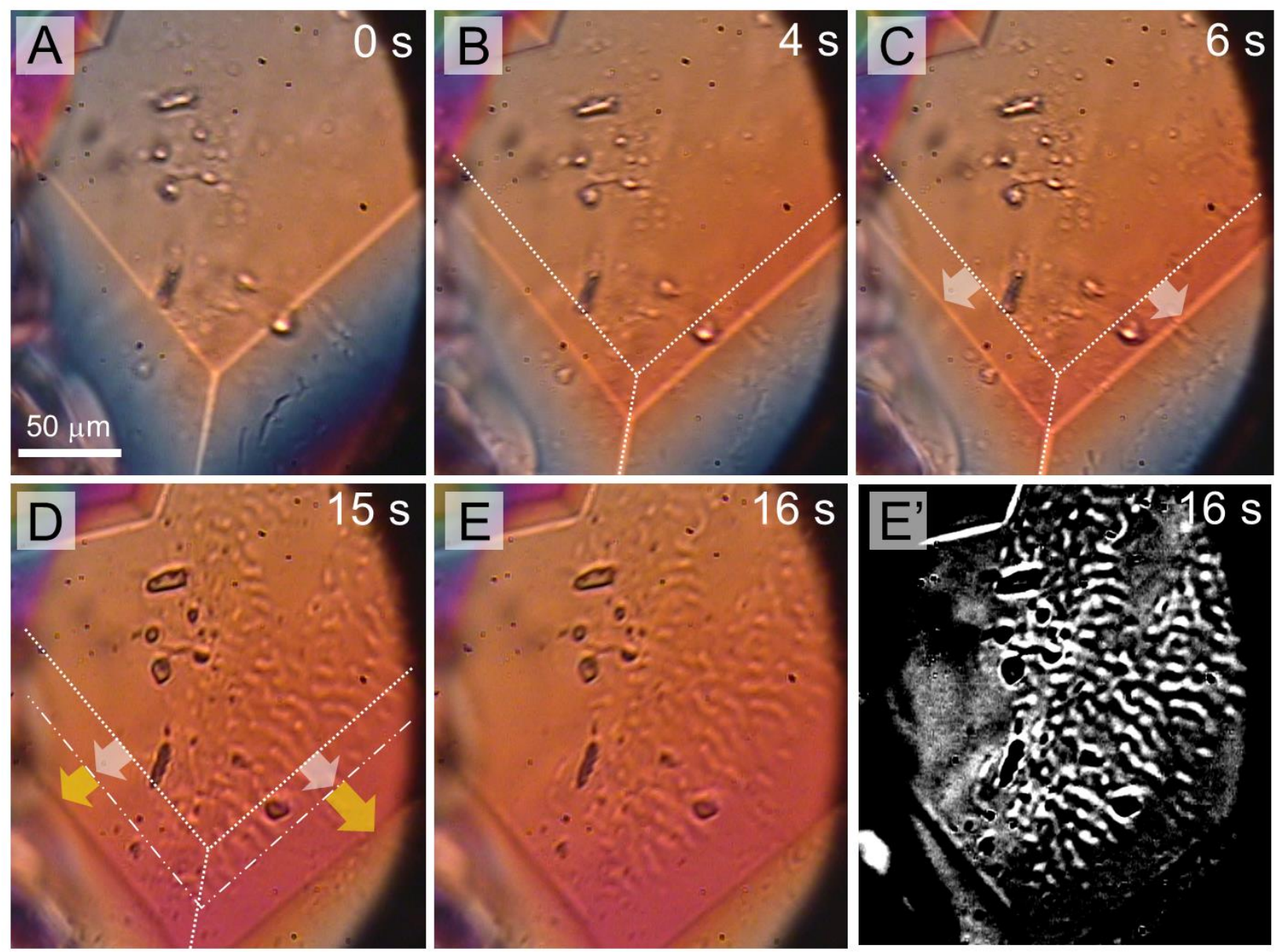

Figure. S4. Measurement of the wavelength of the adhesion-induced fingering instability of HDLL on a growing ice III crystal. (A)-(E) In situ polarized-light photomicrographs showing the emergence of the adhesion-induced fingering instability in the HDLL on the ice III crystal surface (SI Movie S4). (A)-(C) The faceted ice crystal first grew by compression while the HDLL retained a smooth surface morphology. The dotted lines indicate the previous position of the ridge of the crystal. The white arrow indicates the advancement of the crystal ridge from its previous position. (D) An instability pattern began to appear when the HDLL reached a significant distance from the roof of the cell. The dashed-two dotted line indicates the position of the crystal ridge in micrograph $\mathbf{C}$. The yellow arrows indicate the advancement of the crystal ridge. (E) The wavelength of the pattern shown in micrograph $\mathbf{E}$ was measured to be $8.4 \mu \mathrm{m}$. (E') Monochrome version of micrograph $\mathbf{E}$ to improve the visibility of the instability pattern. 

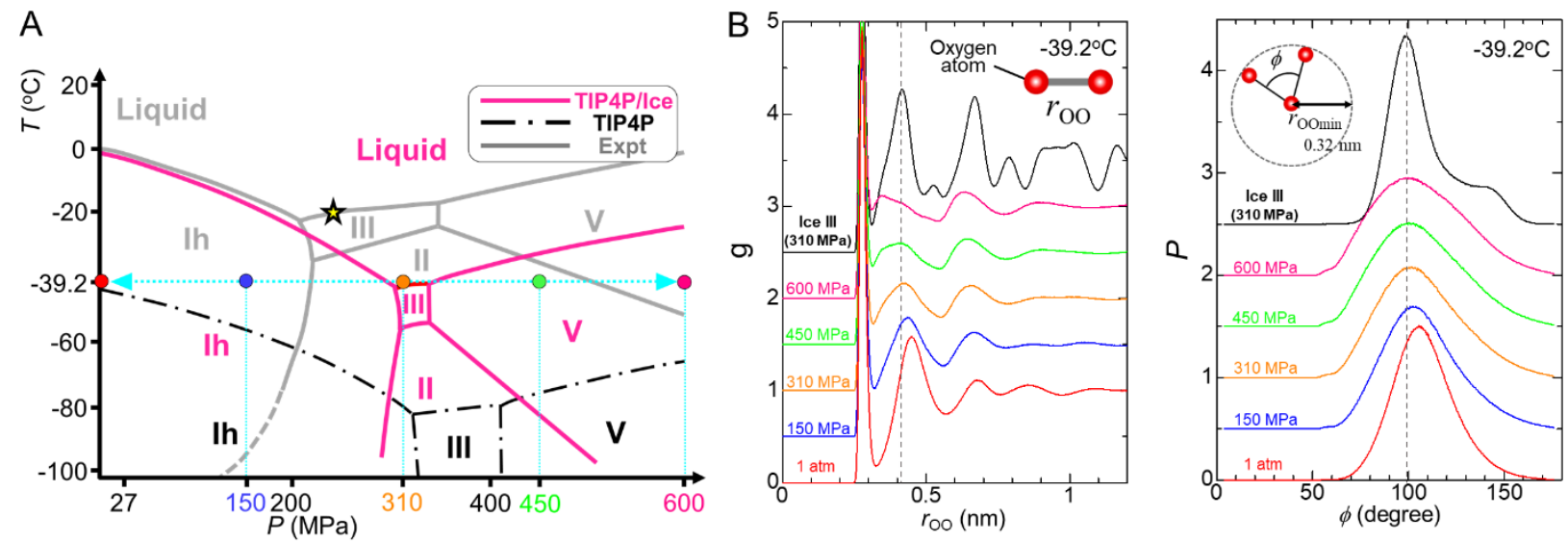

Figure S5. Analysis of the liquid structure of the water under ice III-liquid two-phase coexistence conditions, based on MD numerical simulation. (A) Phase diagram from the TIP4P/Ice model ${ }^{3}$. The magenta lines indicate the phase diagram based on the TIP4P/Ice model, whereas the black dotted lines indicate the phase diagram based on the TIP4P model $^{7}$ and the grey lines show the phase diagram based on experiments. The yellow star indicates the experimental conditions for our in situ observation. The cyan arrow indicates the temperature conditions under which the MD simulations were performed. The red, blue, orange, green, and pink dots represent the temperature and pressure conditions under which the MD simulations were performed: the corresponding pressures were $1 \mathrm{~atm}$ and 150, 310, 450, and $600 \mathrm{MPa}$, respectively. (B) The pair-distribution function $(g)$ for the $\mathrm{O}-\mathrm{O}$ distance $\left(r_{\mathrm{OO}}\right)$ and the distribution function $(P)$ of angle $\phi$ formed by three nearestneighbouring oxygen atoms ${ }^{7}$. The black spectrum is for an ice III crystal at $310 \mathrm{MPa}$. The red, blue, orange, green, and pink spectra are for liquid water at 1 atm and 150, 310, 450, and $600 \mathrm{MPa}$, respectively. roomin indicates the radius referred to by the distribution function. The value of roOmin was set to $0.32 \mathrm{~nm}$ to take into consideration the nearestneighbour oxygen atoms. The vertical grey dotted lines in the $g$ and $P$ diagrams indicate the values of roo and $\phi$ with the maximum peak intensities for the ice III crystal, respectively. The maximum peaks in the roo and $\phi$ distributions at $310 \mathrm{MPa}$ are almost identical to that for the ice III crystal. This suggests that the structure of the liquid water becomes similar to the crystal structure of ice III under the conditions where the two phases coexist. 


\section{Legends for SI Movies S1-S4}

SI Movie S1 (separate file). In situ observation of emergence of a thin liquid layer and droplets distinguishable from bulk water on a surface of an ice III crystal by polarized-light microscopy. The playback speed is real time. Figure 2 consists of snapshots from this movie.

SI Movie S2 (separate file). In situ observation of emergence of a thin liquid layer at the interface between ice VI and bulk water by optical microscope with a Fizeau interferometer. The playback speed is double. Figure 3 consists of snapshots from this movie.

SI Movie S3 (separate file). In situ observation of change in the pattern of HDLL depending on the compression or release of compression, and bicontinuous pattern formation by an optical microscopy. The playback speed is real time. Figure 4 consists of snapshots from this movie.

SI Movie S4 (separate file). In situ observation of adhesion-induced fingering instability of HDLL on an ice III crystal by polarized-light microscopy. The playback speed is real time. Figure S4 consists of snapshots from this movie. 


\section{SI References}

1. Chaudhury, M. K.; Chakrabarti, A.; Ghatak, A. Adhesion-induced instabilities and pattern formation in thin films of elastomers and gels. Eur. Phys. J. E: Soft Matter Biol. Phys. 2015, 38, 82.

2. Chung, J. Y.; Kim, K. H.; Chaudhury, M. K.; Sarkar, J.; Sharma, M. Confinementinduced instability and adhesive failure between dissimilar thin elastic films. Eur. Phys. J. E: Soft Matter Biol. Phys. 2006, 20, 47-53.

3. Abascal, J. L. F.; Sanz, E.; Fernádez, R. G.; Bega, C. A potential model for the study of ices and amorphous water: TIP4P/Ice. J. Chem. Phys. 2005, 122, 234511.

4. Allen, M. P.; Tildesley, D. J. Computer Simulation of Liquid, Clarendon Press, 1987

5. Berendsen, H. J. C.; Postma, J. P. M.; van Gunsteren, W. F.; DiNola, A.; Haak, J. R. Molecular dynamics with coupling to an external bath. J. Chem. Phys. 1984, 81, 3684 .

6. Smith, W.; Forester, T. R. DL_POLY 2.0: A general-purpose parallel molecular dynamics simulation package. J. Mol. Graphics 1996, 14, 136.

7. Tomono, H.; Nada, H.; Zhu, F.; Sakamoto, T.; Nishimura, T.; Kato, T. Effects of magnesium ions and water molecules on the structure of amorphous calcium carbonate: A molecular dynamics study. J. Phys. Chem. B 2013, 117, 14849. 